\title{
Intervention of Sleep Quality in the Onset of Concomitant Symptoms in Hospitalized Patients
}

\author{
Gabriel Miranda Nava* \\ Head of the Neurology service, Regional military Hospital of specialties of Guadalajara, Mexico
}

*Corresponding author: Gabriel Miranda Nava, Head of the Neurology service, Regional military Hospital of specialties of Guadalajara, Mexico.

Received Date: June 10, 2019

Published Date: June 13, 2019

\begin{abstract}
This work allows us to observe the quality of sleep and the respect of circadian cycles as very important factors in the development of multiple diseases and in various specialties, which affects the methods Therapeutic, both in outpatient and in hospitalized and the variety of symptoms that can be observed, through a method of surveys in a practical way, with the main objective of contributing in the Awareness to treating physicians about the importance of sleep hygiene measures and their improvement in patients quality of life. This document aims to fulfil the quality of sleep and the circadian respect cycles as important factors in the development of multiple diseases in various specialties, which affects therapeutic methods both in outpatients and in hospitalized ones, and the variety of symptoms, that can be observed, through a practical survey method, with the main objective of contributing to the awareness of treating physicians on the importance of sleep hygiene measures, and improving patients quality life.
\end{abstract}

\section{Introduction}

When trying to define the dream it is in evi Figure to differentiate the physiological characteristics in the good quality of the sleep depending on the species, where it is a little easier to enumerate the behavioral characteristics that are associated with the aspect of the sleep and Trying to define it properly, where it can be associated with immobility and muscle relaxation, this is a reversible event (which differentiates it from other pathological states such as stupor and coma), decreased consciousness and reactivity to external stimuli and finally during sleep individuals acquire a stereotyped posture. The absence of sleep induces different behavioral and physiological alterations in addition to generating a cumulative debt of sleep that eventually must be recovered. A tool that has been of vital importance to the study of the physiology of the Sleep is THE electroencephalogram (EEG), which is the graphic and digital representation of the oscillations that shows the electrical activity of the brain, When they are registered by electrodes placed in different regions of the head. During alertness while keeping eyes closed on THE EEG are observed oscillations of the electrical activity that are usually between 8-13 cycles per second, mainly in occipital regions (alpha rhythm) within the sleep changes occur characteristic of the brain activity that are the basis for dividing the sleep into several phases, where it is usually divided into two phases: No eye movements Quick, and with eye movements Quick.

Dream NO MOR: Phase n1 corresponds to drowsiness or the onset of light sleep, it is often observed slight muscular jolts (sharp vertex waves), phase $\mathrm{n} 2$ appear specific patterns of Brain activity, called sleep spindle and $\mathrm{k}$ complex, the cardiac and respiratory frequency begins to decrease paulativamente, Phase N3 or slow wave sleep is the Deepest phase is observed in The EEG very slow frequency activity $(<2 \mathrm{~Hz})$. The dream MOR: now called the R-phase and is characterized by the presence of rapid eye movements, physically all muscle tone decreases. A young adult spends approximately 70-100 min in Sleep no MOR which can last between 5-30 min and this cycle is repeated every hour and a half throughout the night, therefore throughout the night can normally be presented between 4 and 6 cycles of sleep MOR.

\section{Sleep hygiene}

Sleep hygiene measures are a series of recommendations on desirable behaviors and habits, as well as changes in environmental conditions and other related factors aimed at improving the quality of Dream of people who already suffer from a sleep disorder such 
as insomnia, or that can be used as measures to prevent a sleep disturbance. Although there is no global consensus on what These sleep hygiene measures should be and some of these sleep hygiene measures are transplanted with some forms of general non-pharmacological behavioral treatment We can consider (avoid prolonged naps $>1 \mathrm{hr}$, lie down to sleep at the same time every day, wake up at the same time all the way, sleep in comfort Figure bed, sleep in a room with low lighting and little ambient noise).

\section{Epidemiology}

It is estimated that, in our country, about $45 \%$ of the adult population has poor quality of sleep. The above is reflected in the difficulty that people have to get up, as well as in constant drowsiness and fatigue during the early hours of the morning.

\section{Materials and Methods}

The present study uses the reference method, cionandoencues Tas descriptive, and Testing the Pittsburgh sleep quality scale where 20 patients in hospital are assessed in the Hospital MIlitar Regional E Specialties of Guadalajara, Jalisco, valuing the level of sleep quality and Quéconcomitant symptoms are added by poor sleep hygiene, probationary period February-May.

\section{Justification}

This research will focus on studying hygiene habits based on sleep quality in hospitalized patients, because due to occupational stress or hospital instances it has been seen that patients have directly modified their sleep habits Worsening quality of life and delaying hospital recovery by increasing concomitant symptoms, we then propose to investigate the evolution of pathological patterns and their aggregate symptoms ; the reasons that led us to investigate the quality of sleep in hospitalized patients are due to increased discomfort or data from patient-referred drowsiness after staying more than 2 days hospitalized. Pensamors that through the detailed analysis we can raise awareness of health personnel to improve the hygiene of the Dream so help the prompt recovery In appearance of concomitant symptoms in patients who encu They're in hospital.

\section{Objectives}

1. To observe clinically the behavior of the hospitalized patients and their quality in the hygiene of the sleep.

2. Intervene and prevent concomitant pathologies that are associated with poor hygienic sleep habits.

3. Improve the quality of life of hospitalized patients.

4. To raise awareness of the application of hygiene or good sleep habits to improve the quality of life.

5. Decrease the percentage of symptoms added by decrease in sleep schedules.

6. Locating key events or outstanding problems in sleep quality.

\section{Statistical Population}

A sampling was carried out where people of both sexes were chosen, who were subjected to a descriptive evaluation by means of direct surveys, where a sample of 20 people with characteristics with a simple random choice type was collected, by making a list of participants hospitalstalizados to form the sample to be studied by means of questionnaires.

\section{Result}

It takes a random sample to conduct direct descriptive surveys in the Hospital Militar Regional of Guadalajara, Jalisco, where They take 14 people of the female sex and 6 people of the male sex, having a percentage of $70 \%$ female and $30 \%$ male. As for the EDAD there is an ambiguous percentage in which it is observed that $30 \%$ correspond to people with age between 21 and 30 years following in a proportional way to $15 \%$ patients D and age 31 to 73 years. (Figure 1). In the marital statusthe $74 \%$ of respondents are married, $11 \%$ are single, and $15 \%$ proportionally are divorced, widowed and in Union Free (Figures 1,2).

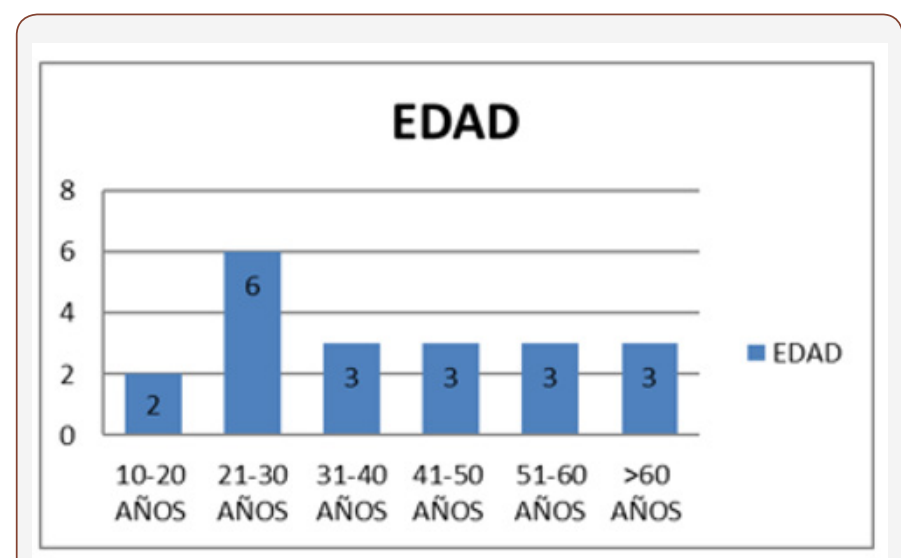

Figure 1: Age of the sample patients.

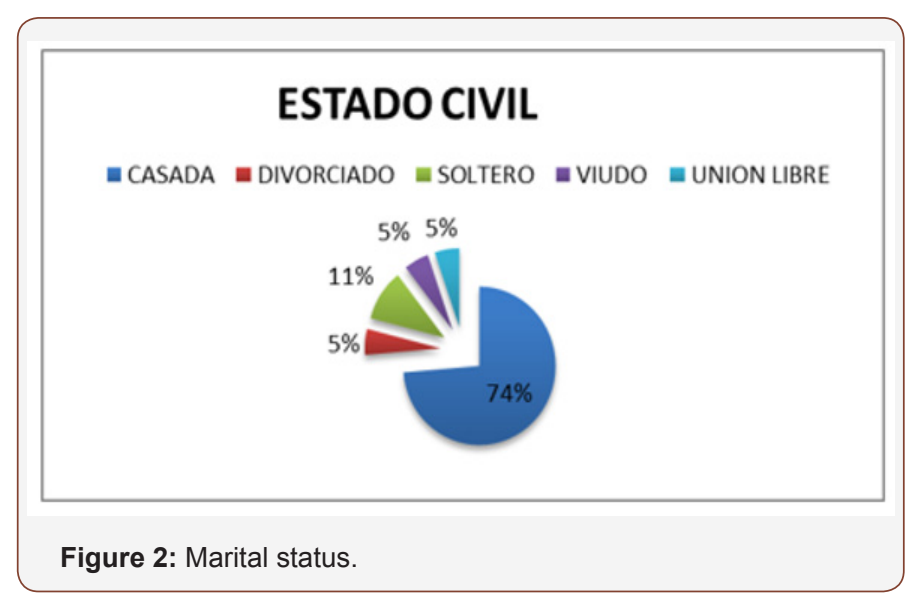

Within the descriptive surveys were not taken into account the situations of Derechohabiencia in the institution where the majority of reported or interviewed were successors in the asset having the majority of percentage with a $60 \%$ followed by military in The active where the majority of the concomitant symptoms corresponded to anxiety and headache (Figure 3). 


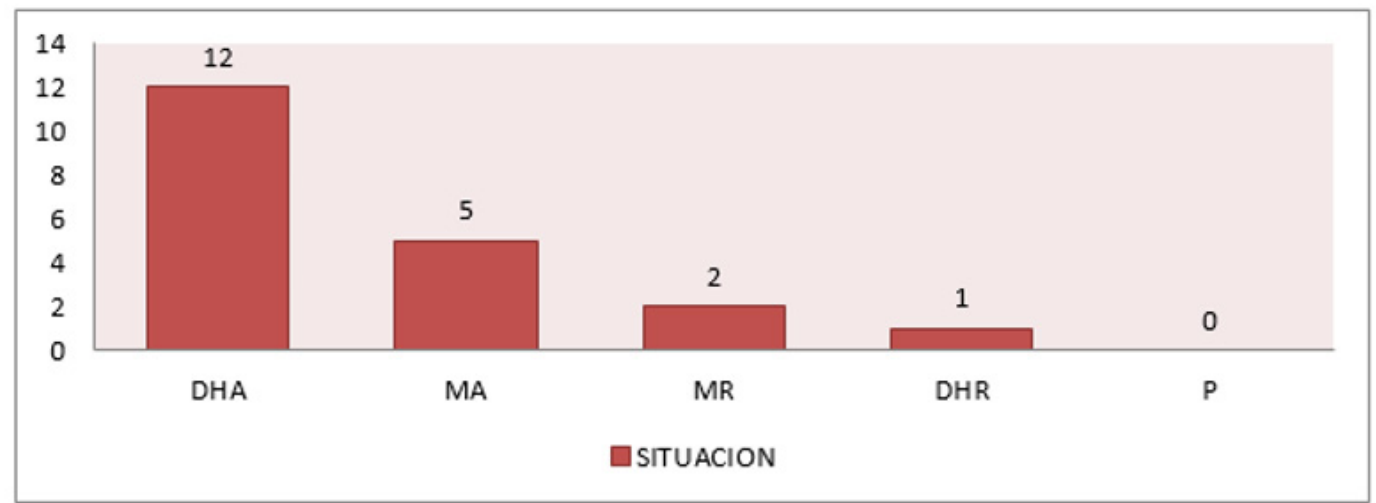

Figure 3: Social security situation.

Among the key questions that are characterized in the Pittsburgh scale, it is time to lie down where literally $50 \%$ of the patients interviewed reiterate or lie down to sleep around 10:00 pm and 11:00 pm, while the remainder was distributed with $25 \%$ which lie regularly from 08:00 pm to 09:00 pm and the Otro $25 \%$ after 12:00 pm (Figure 4).

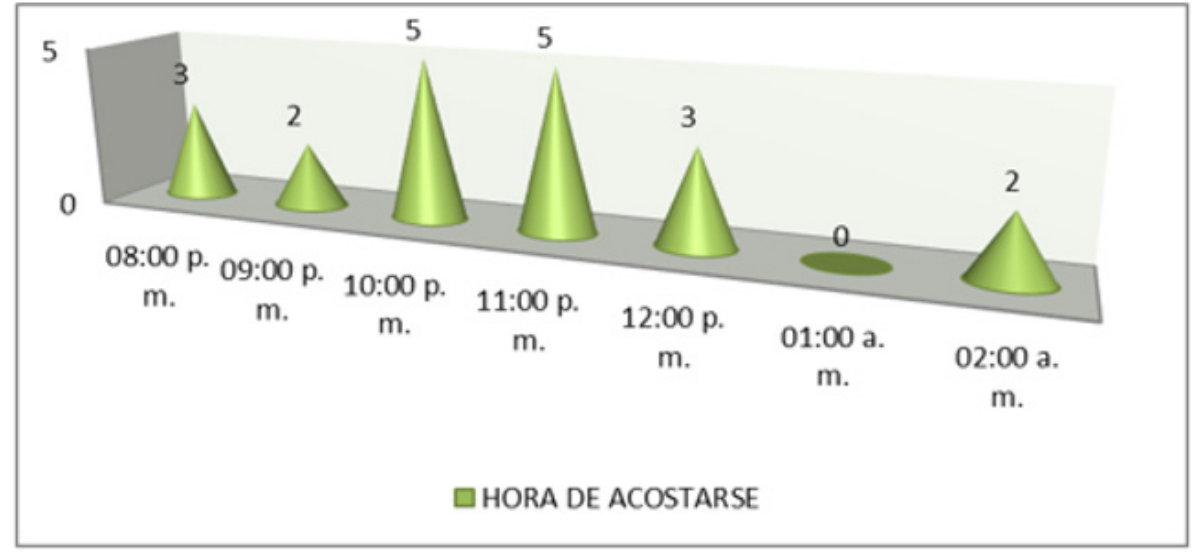

Figure 4: Time to go to bed.

Continuing with the variability of the sleep habits se promptly makes the question about the total time it takes to fall asleep indispensably, where results or a variability Detailed between each point and constant obtaining as a result that $45 \%$ of the population studied sleep within the first 10 minutes, while $25 \%$ is distributed in patients who sleep within the first $30 \mathrm{~min}$ and a data Important is that $15 \%$ of patients sample lasts more than 60 minutes to fall asleep (Figure 5).

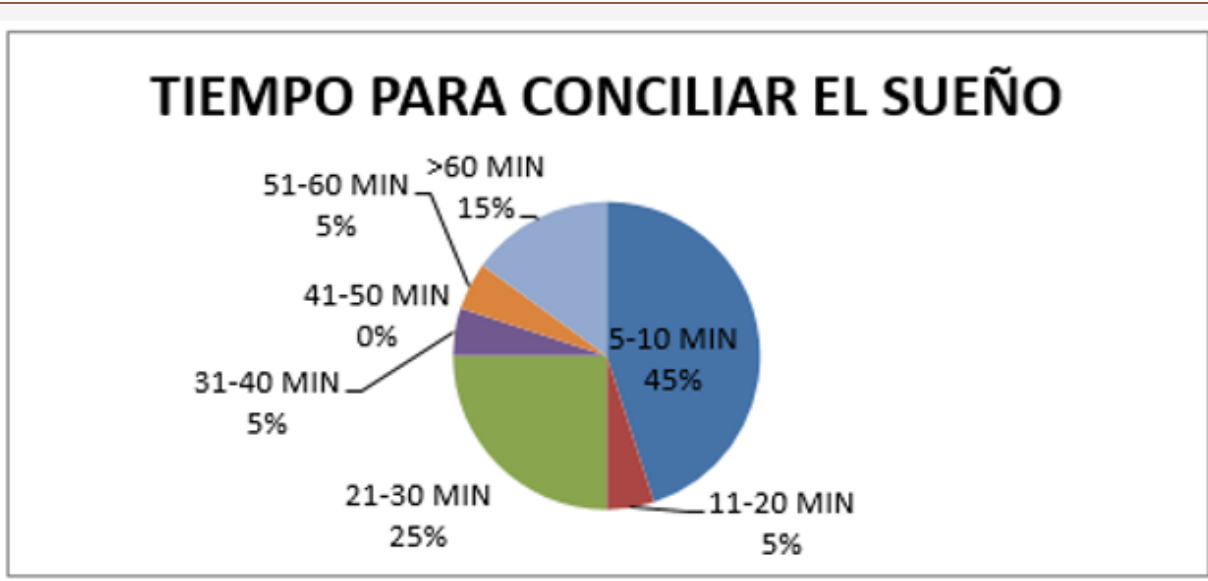

Figure 5:Sleep reconciliation time (sleep latency).

It should be noted the relevance the time it takes people to sleep and the schedule they have to get up, as it should be noted that within the quality of sleep sand knows that a young adult or should
Sleep an average of 7-8 hours, although this amount may vary because it depends on internal factors of the organism and external of the Society, therefore a preschool child can sleep between 11 
or 12 hours and an older adult between 5 and 6 hours not taking into account the per Iodines that slumber during the day, so it is of paramount importance the results in which the percentages of patients who wake up early and sleep late as they begin to have functional and organic damage that are seen Involved in their daily lives. Don the Pittsburgh scale, it is essential to highlight and emphasize the following two patterns:

- $\quad$ The time when patients regularly wake up from their dreams

- $\quad$ The total hours of sleep of a patient.

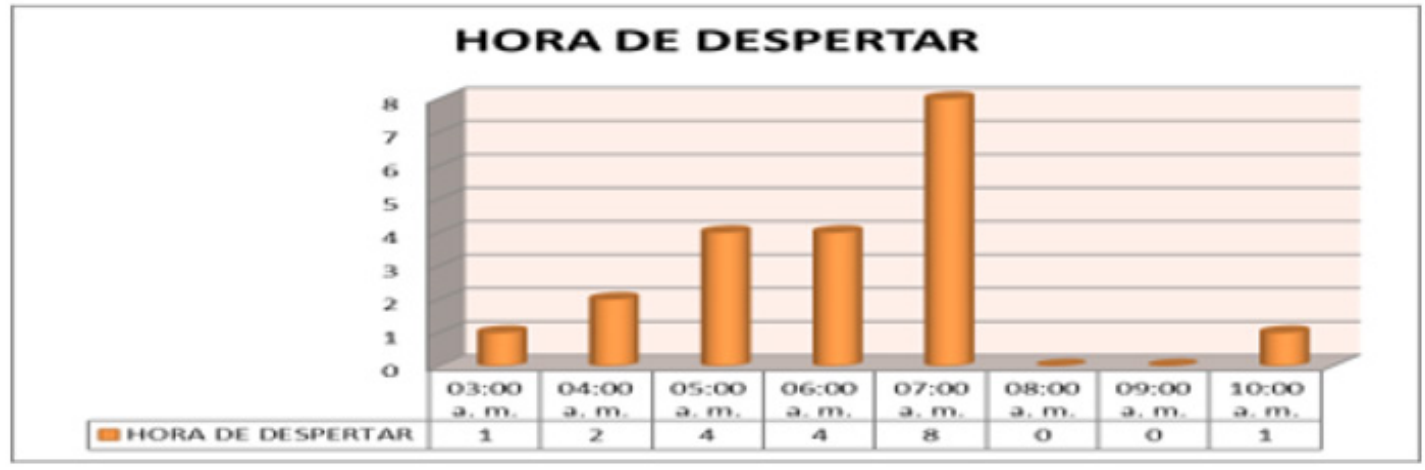

Figure 6:Average waking time.

In Donde the results having as supremacy that $40 \%$ of the population studied awake at 7:00 am while the other $40 \%$ awake on an average between 5:00 and 6:00 am to perform their Activities, finally $15 \%$ have a short average sleep wake between 3:00 and 4:00 am (Figure 6).

Performing and observing an average within the sleep habits and the quality of sleep of the patients is asked a question to evaluate the number of hours that people sleep a day approximately, taking into account their basic pathologies (PO Stop erated, chronic degenerative pathologies, concomitant pathologies), having as a result that $45 \%$ of patients or sleep 5 to 6 hours in a row compared with the schedule that a person should actually sleep to refer A proper sleep quality and hygiene is really low in established parameters, while about $15 \%$ of patients taken as a sample sleep an average of 10 to 15 hours a day, it should be noted that these patients mentioned Having psychological and psychiatric problems as a depression, if we recap the concepts offered by the National Dream Foundation, $85 \%$ of the patients interviewed do not meet the criteria to define that they maintain adequate sleep health, while That only $5 \%$ of patients could enter the criteria already established and mentioned (the following graph shows the number of patients and the total sleep hours they maintain for one day) the Pittsburgh scale There are questions to be mentioned that are subjective processes that take relevance in the last 4 sem Anas, about falling asleep where $45 \%$ of hospitalized patients contest or that approximately three times a week are prevented from reconciling sleep in a proper way. And evaluating the number of people and times approximate mind rise during the nights were obtained results that 14 people of the interviewees rise between 2 to 3 times per week which is about $70 \%$ of patients surveyed with those bad habits of sleep. With regard to the reasons why the patients were awakened the following result was taken Figure 7 \&8).

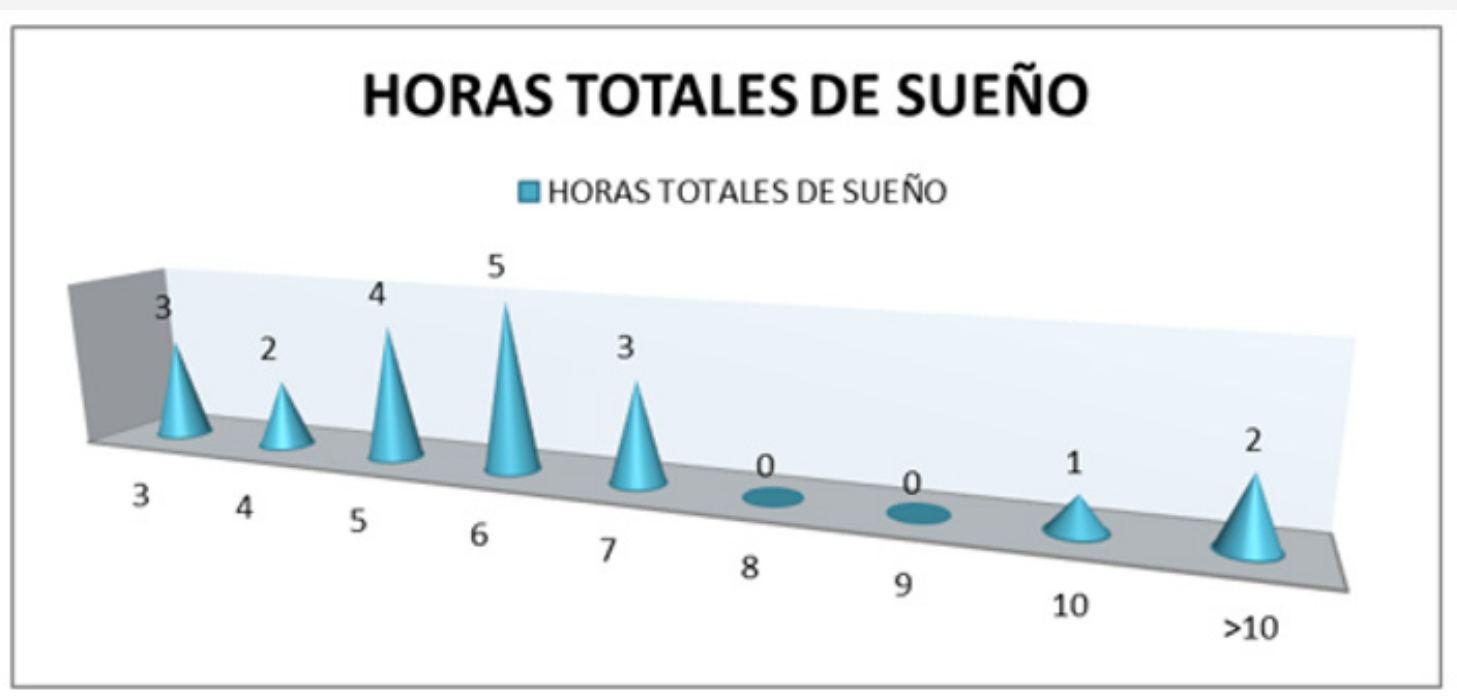

Figure 7 


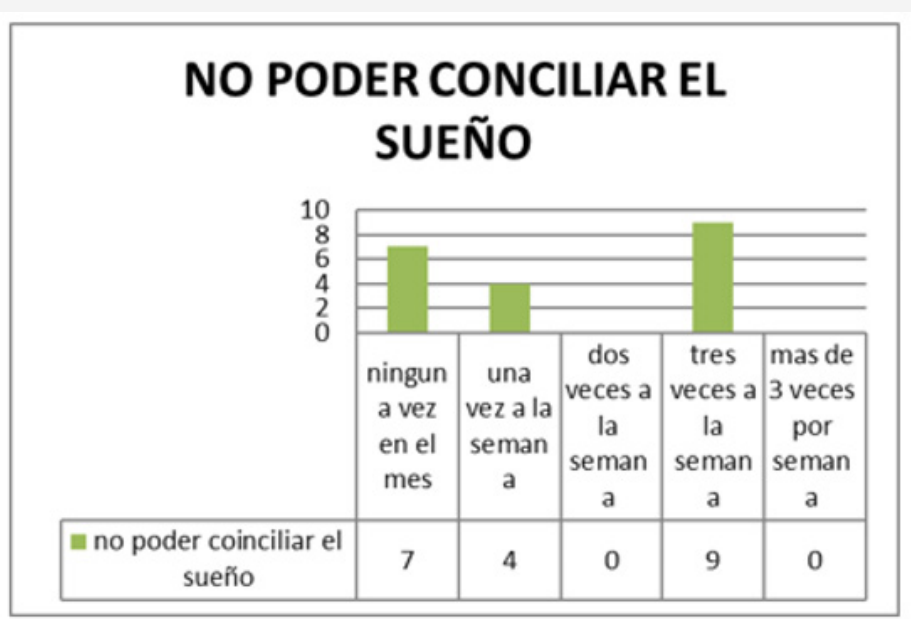

Figure 8

Where $65 \%$ of respondents in the last month would not have suffered from feelings of lack of air, while the number of 6 people reported that three times in the week are awakened by feeling a lack of Air corresponding to $30 \%$ of the sample investigated (Figure 9).

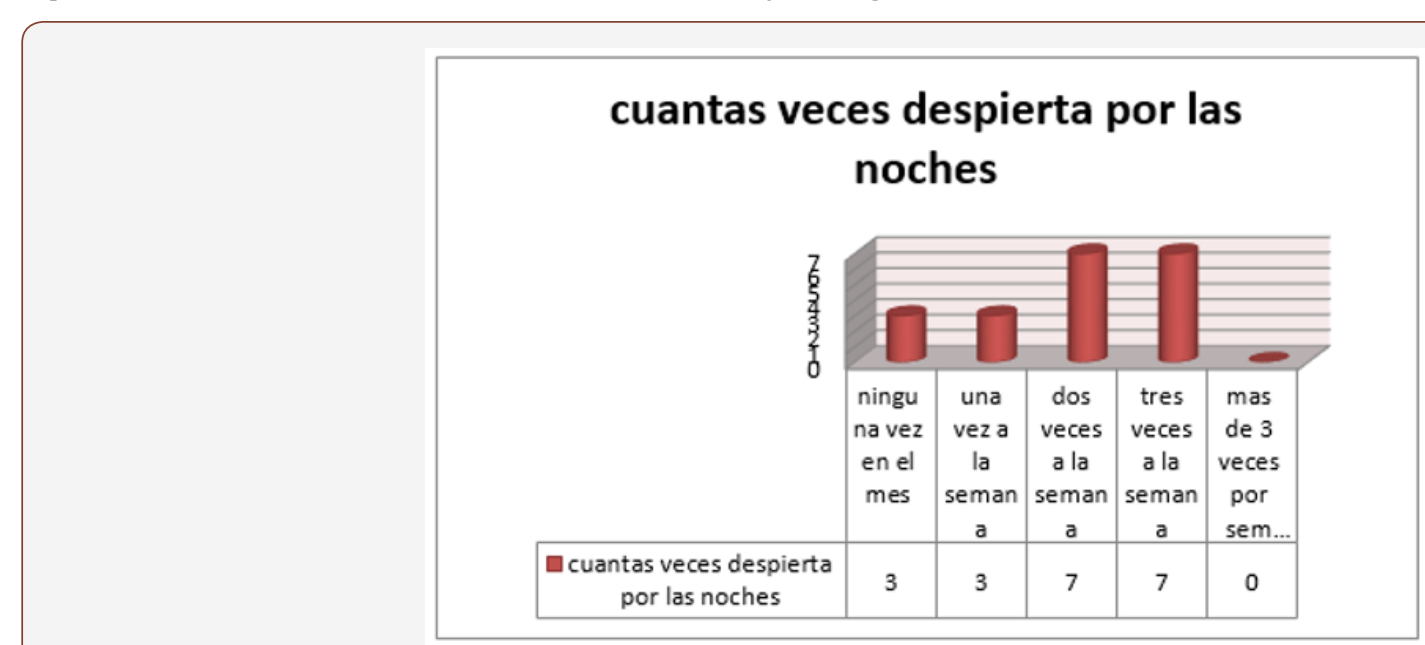

Figure 9

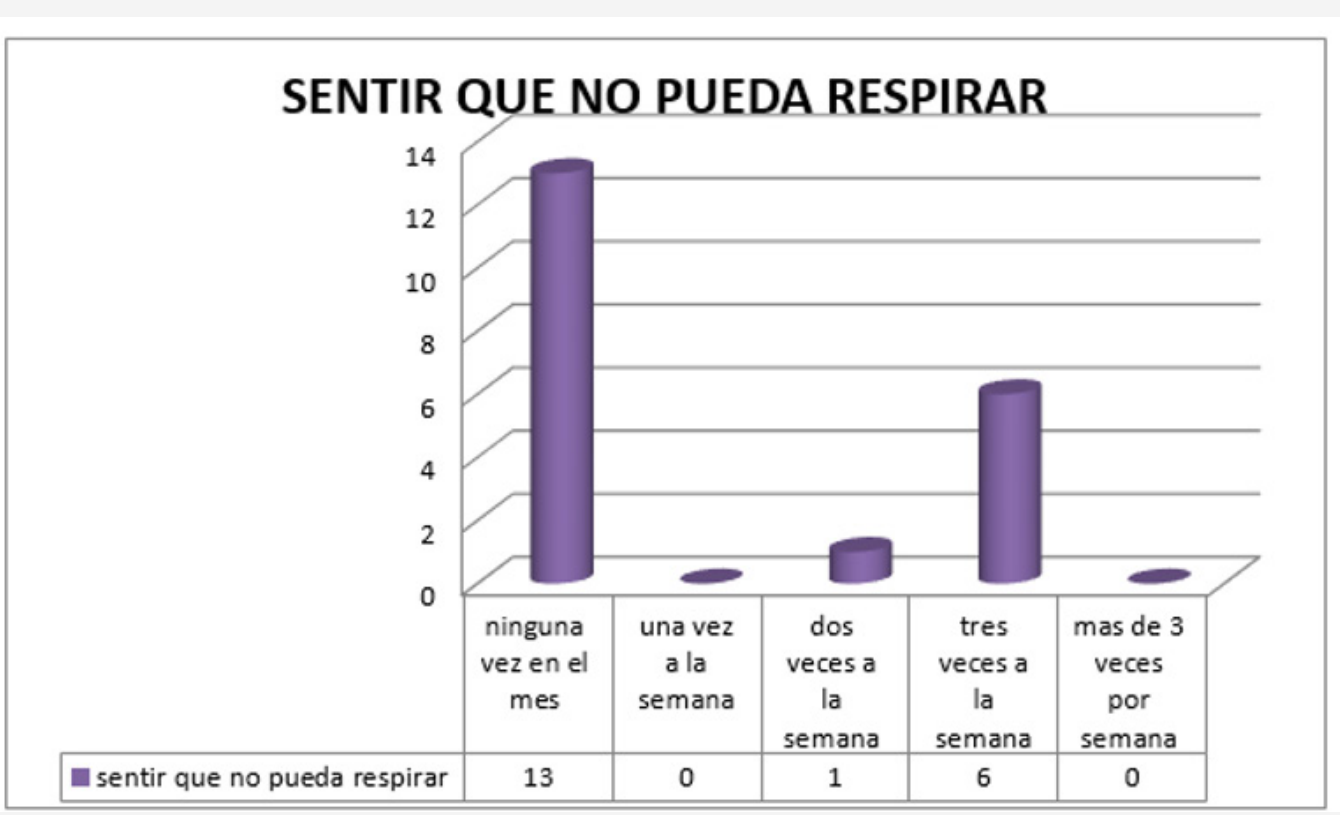

Figure 10 
When asking indirect questions about whether your family member snored $40 \%$ Contest or did not capture such events in the last 4 weeks while $45 \%$ responded to that on an average of 1-2 times per week snoring at night what That made the patient wake up during the night, $50 \%$ of patients have not woken during the night for the last 4 weeks, and with a total of 5 patients it results $25 \%$ that they woke up at least once a week for feeling of Frio. In the opposite case, $70 \%$ of the patients Sun would wake up during the night by feeling of warmth (Figure 10-15).

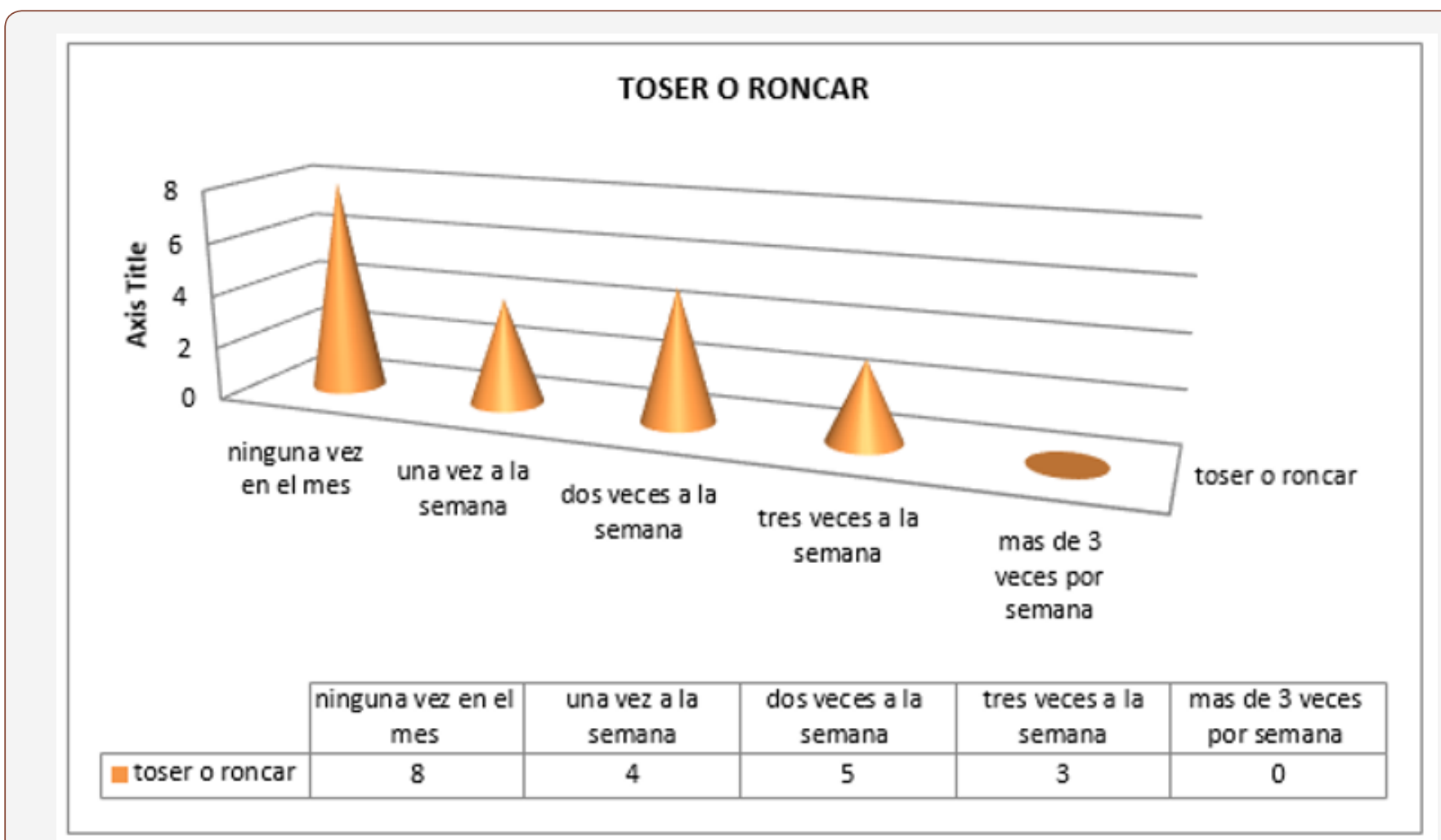

Figure 11

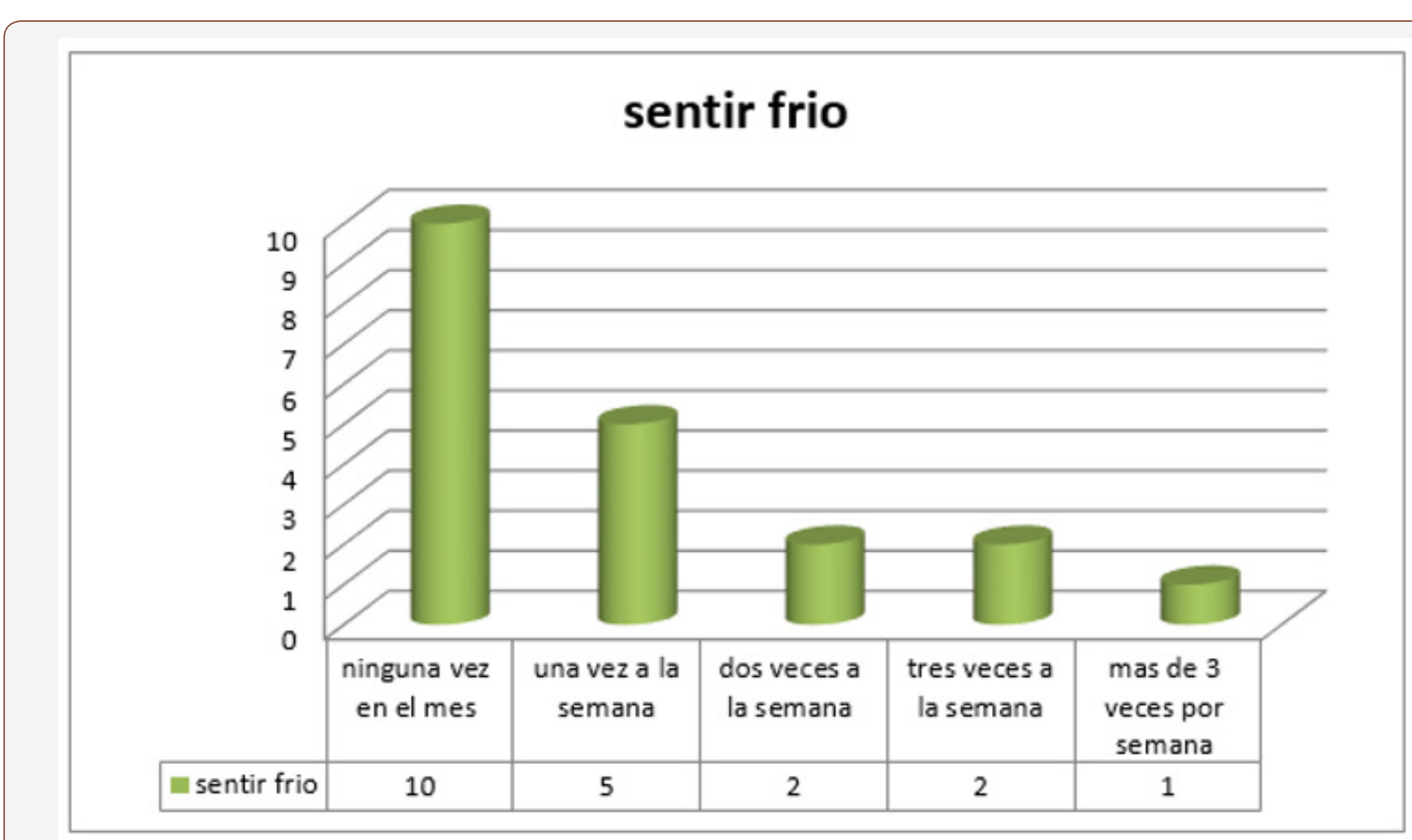

Figure 12 


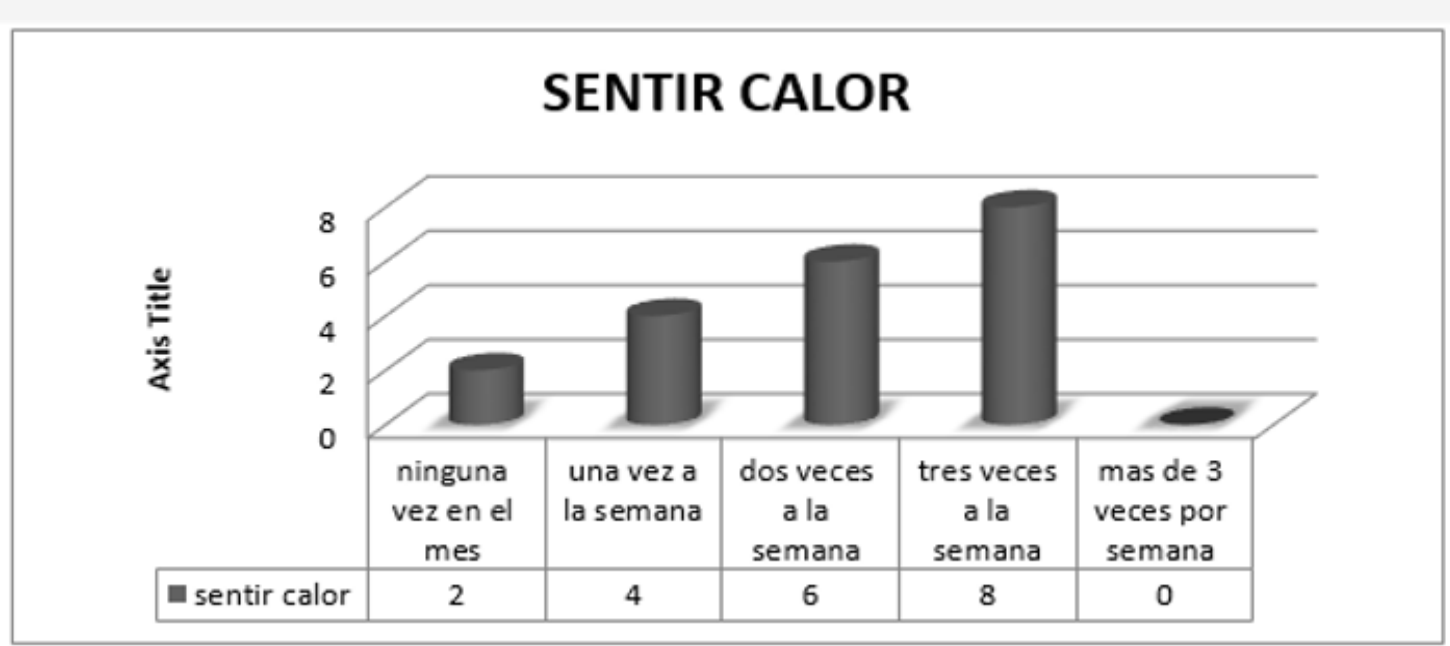

Figure 13

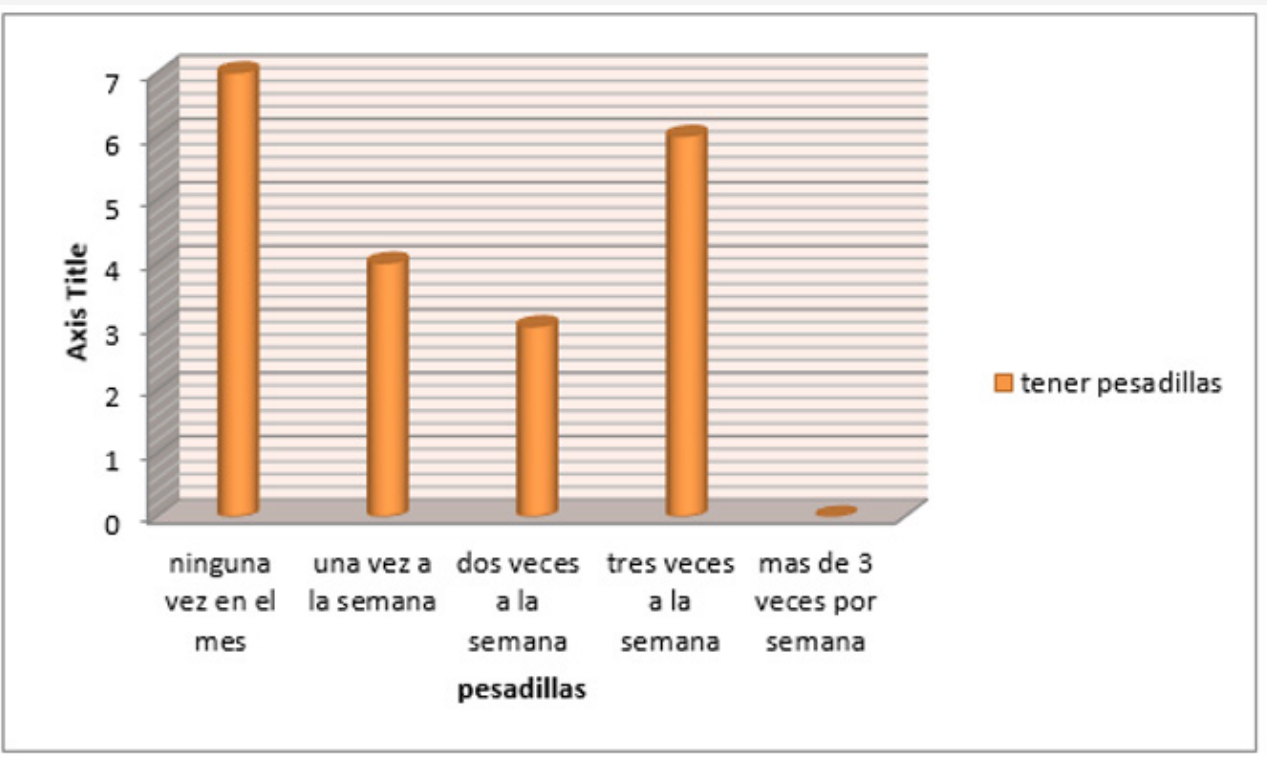

Figure 14

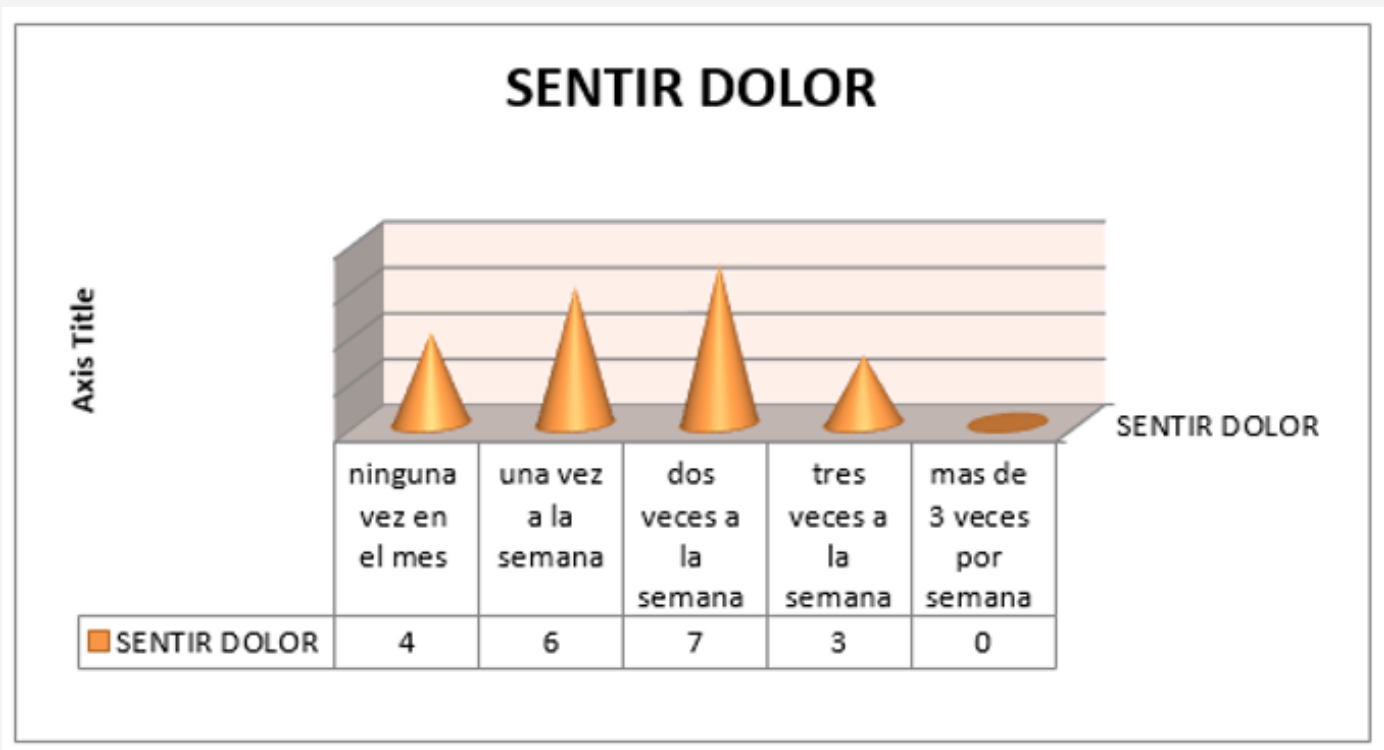

Figure 15 


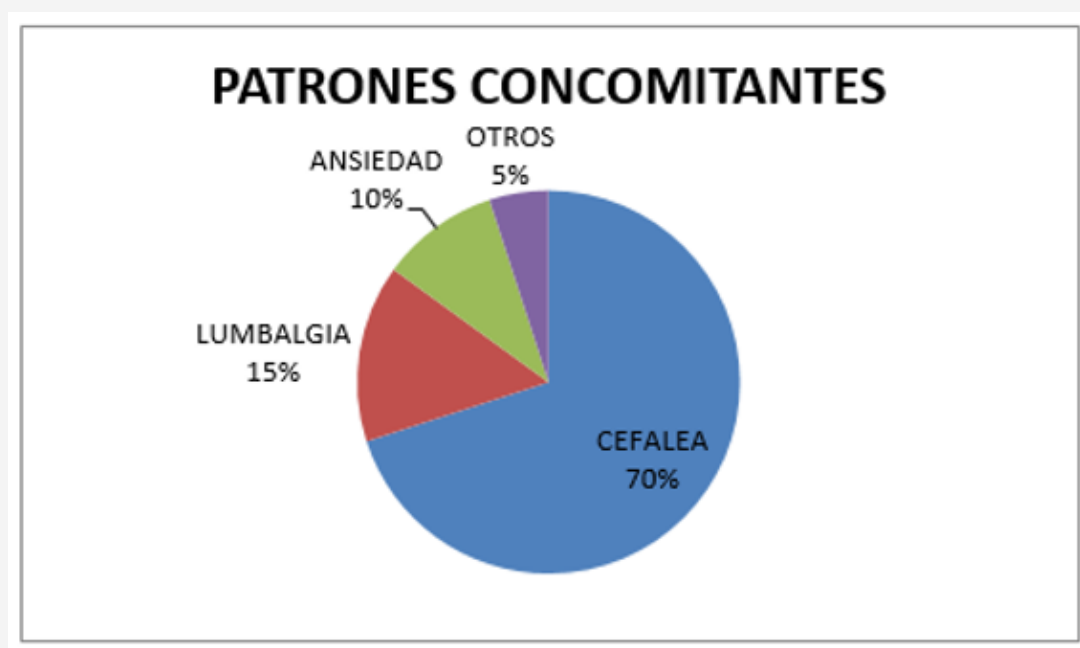

Figure 16

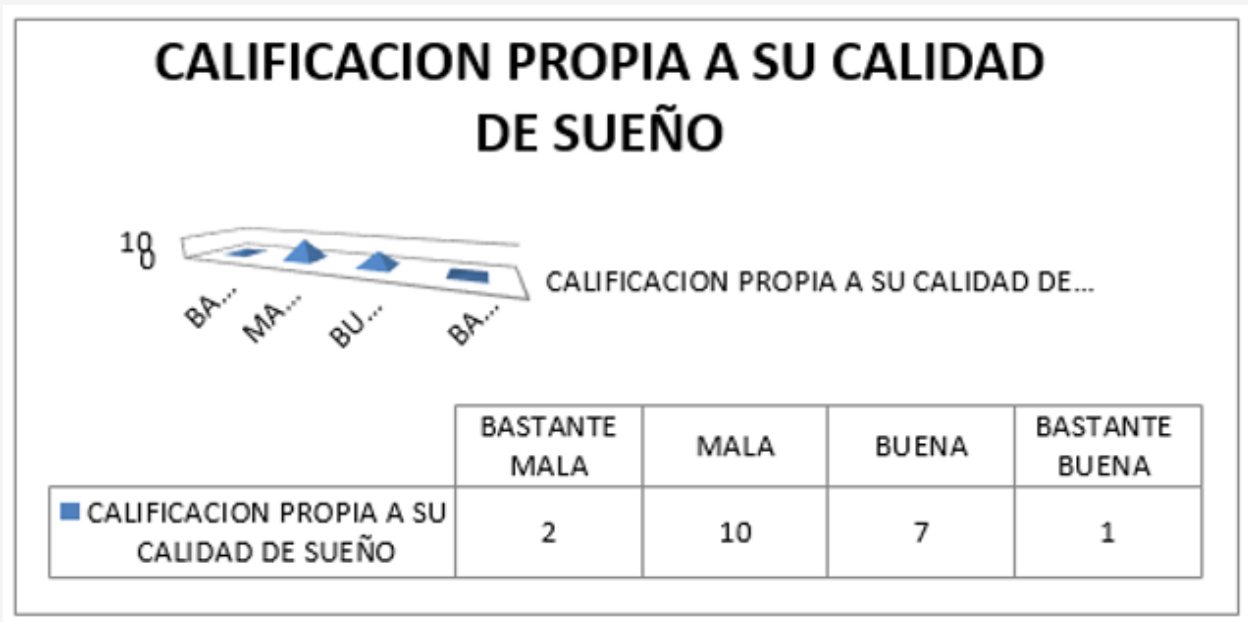

Figure 17

\section{USO DE MEDICAMENTOS PARA COINCILIAR EL SUEÑO}

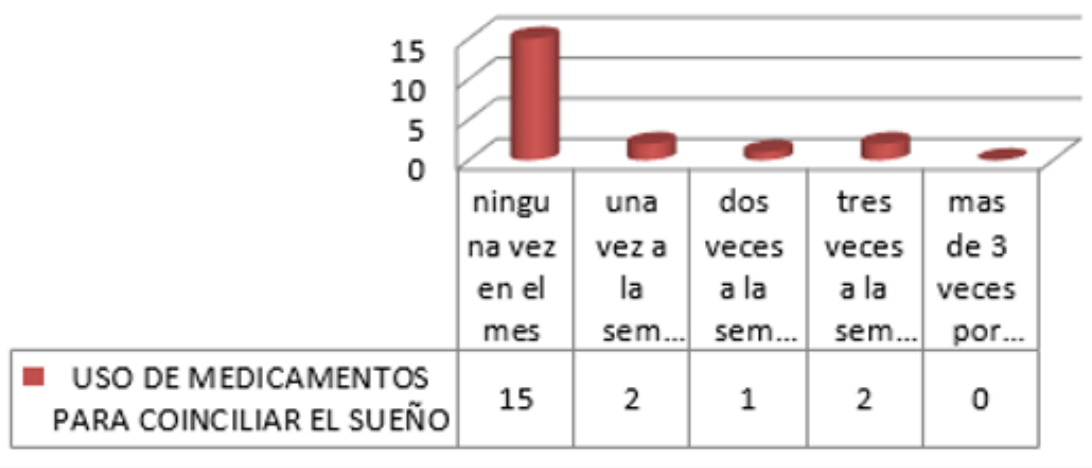

Figure 18

$35 \%$ of the patients surveyed resulted in no problems such as nightmares at bedtime, while the other 35\% have nightmares 1 or 2 times per semana, $30 \% 3$ times per semana; the next GR to FICA is representative of the results obtained by applying that question to the sample that was investigated. Within the data you want to highlight in this study is the presence of pain and What type in the last 4 weeks, referring to pain as an event that keeps them awake or wake them during the night, Having as a conclusion 
that $30 \%$ of the patients interviewed answered or affirmatively that approximately once a week felt pain, and the 35\% mentions or that approximately twice a Week I felt some kind of pain. When evaluating Paulativamente The data provided by patients was obtained as a result that $70 \%$ of hospitalized patients have presented tension headache caused by the bad Háhabits or Sleep hygiene, adding low back pain and even anxiety in a total of 5 patients more that correspond another $25 \% \mathrm{~m}$ to $\mathrm{S}$ of the sample studied (Figure 16-21).

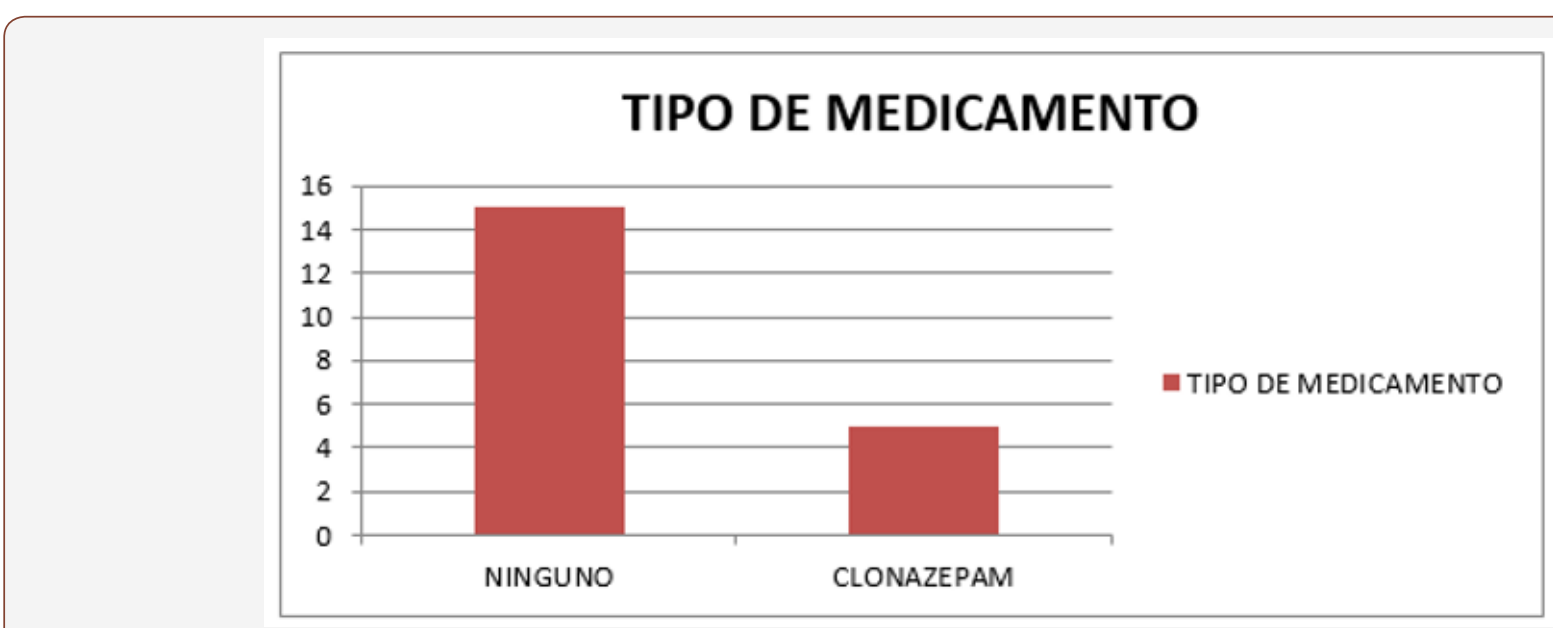

Figure 19

\section{SENSACION DE SOMNOLENCIA}

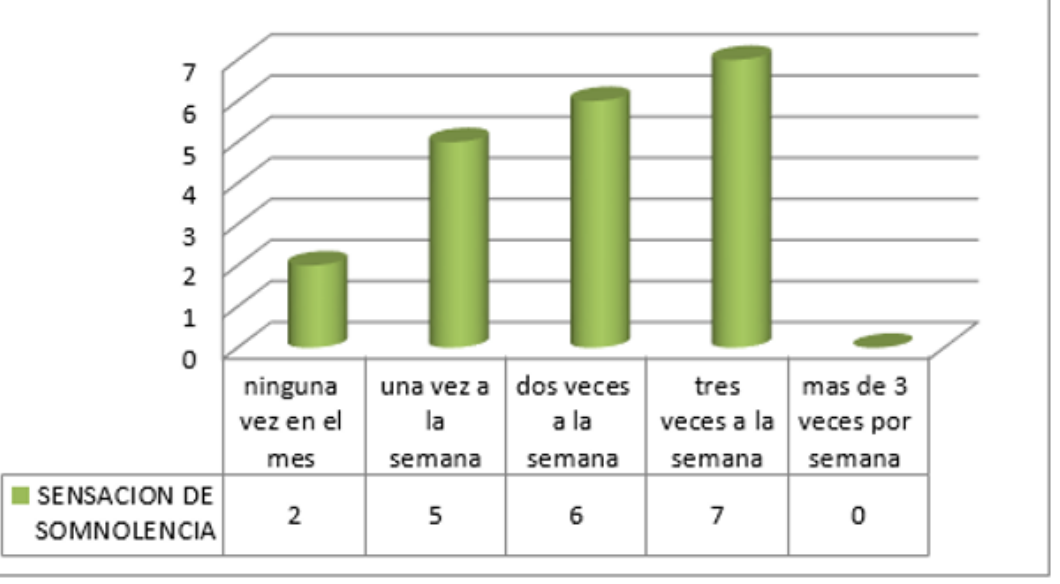

Figure 20

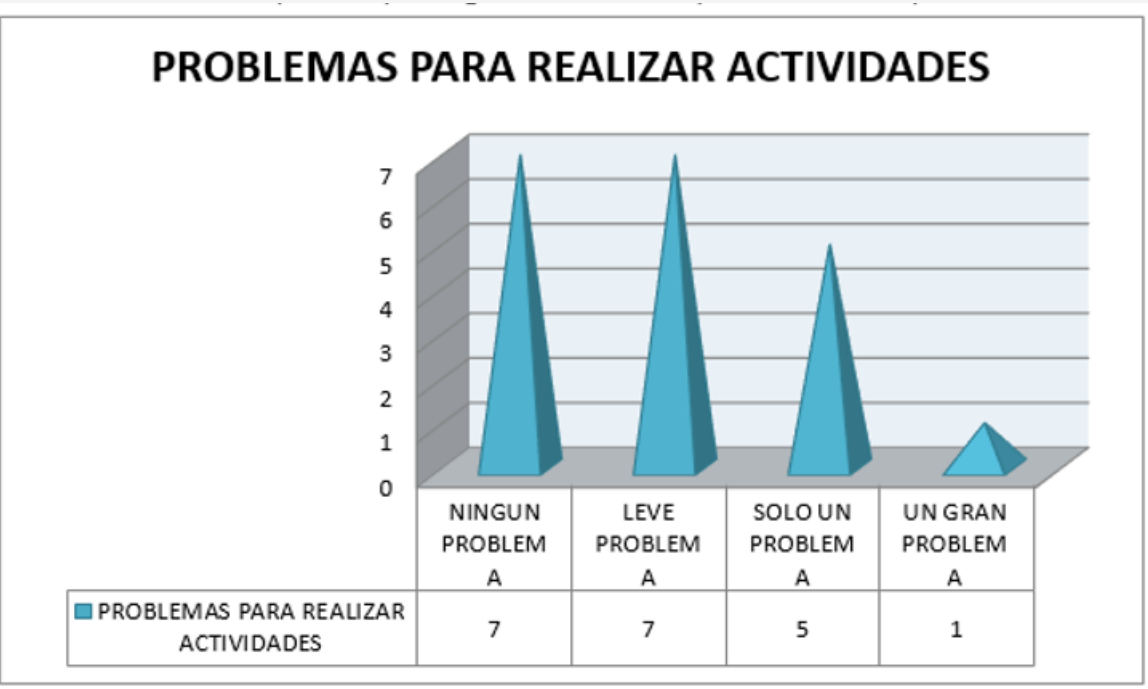

Figure 21 
El $50 \%$ of the patients caliciform their quality of sleep as bad whereas $35,1 \%$ the caliciform like a good dream, you will be asked or taken any medication or taken in the last 4 weeks to be able to fall asleep, where $75 \%$ denied having taken medicine to fall asleep. While the other $25 \%$ acceptor having ingested medicine to be able to sleep either 1 to 3 times a week. The unanimous drug that $25 \%$ of the patients used is clonazepam which is a benzodiazepine that acts directly in the central nervous system, which can create drowsiness and fatigue. In the study of sleep hygiene there is variability where it focuses directly on factors such as drowsiness that is directly caused by poor hygiene habits in sleep, based on the scale of Pittsburgh There is a direct section where it makes questions about the drowsiness that the patient could suffer and finally if that drowsiness or tiredness could prevent the accomplishment of some daily activity, or executive in the Day to Day.

Having as a result that $65 \%$ of patients have been found in a status of drowsiness or have felt tired in an approximate period of between twice to three times a week, while Sor $25 \%$ of patients You have drowsiness at least once a week, preventing your early recovery or decreasing your stamina in everyday life.

Taking in cue NTA the number of patients who responded says TiVo to the question about drowsiness was ob had a graph showing the results in which manifested the inability or problem that originated Maintaining that status of drowsiness obtaining that $35 \%$ of the patients have no problem in carrying out their daily activities, while another $35 \%$ of the people survey ed give or their concern and calcific or as a slight problem in everyday life keeping up with so data mn Olencia which can cause difficulties in your daily life; finally $25 \%$ caliciform their level of drowsiness as a moderate problem by defining thaton Daily Activities Sí It causes them conflict to be able to perform them with the greatest aptitude and energy.

\section{Conclusion}

Definitely the great problem of health to solve in our country has to do necessarily with the habits and because, by not respecting the hours of sleep, alter the biorhythms that translate into clinical problems such as: Headache, nervous colitis, depression, anxiety, metabolic and systemic problems. It is important to understand the re-education to our patients in each consultation and sanitary step, since the installation of an adequate diet and weight control, as well as a healthy and aerobic sport together with the measures of sleep hygiene will be the main weapons for Attacking various diseases in our time.

\section{Acknowledgement}

None.

\section{Conflict of Interest}

No conflict of interest.

\section{References}

1. Lluesma Vidal M, Murgui Pérez S, Carmona Simarro JV (2019) Nutritional status regarding the quality of life and sleep pattern in communitydwelling older adults with cognitive dysfunction. Nutr Hosp 36(2): 303308.

2. Garrigós-Pedrón M, Segura-Ortí E, Gracia-Naya M, La Touche R (2019) Predictive factors of sleep quality in patients with chronic migraine. Neurologia S0213-4853(19): 30014-30023.

3. Serrano-Guzmán M, Valenza-Peña CM, Serrano-Guzmán C, AguilarFerrándiz E, Valenza-Demet G, et al. (2016) Effects of a dance therapy programme on quality of life, sleep and blood pressure in middle-aged women: A randomised controlled trial. Med Clin (Barc) 147(8): 334-339.

4. Abad Massanet F, Rivero Pérez J, Vera Osorio JA (2019) Differences in health-related quality of Life between men and women with sleepdisordered breathing. Semergen 41(8): 407-412.

5. Cruz T, García L, Álvarez MA, Manzanero AL (2019) Sleep quality and memory function in healthy ageing. Neurologia S0213-4853(19)300039.

6. Escobar Fernández L, Coccolo Góngora A, Vázquez López M, Polo Arrondo AP, Miranda Herrero MC, et al. (2019) Continuous spike-waves during slow-wave sleep: Experience during 20 years. An Pediatr (Barc) S1695-4033(19)30021-9.

7. Abbott SM, Weng J, Reid KJ, Daviglus ML, Gallo LC et al. (2019) Sleep Timing, Stability, and BP in the Sueño Ancillary Study of the Hispanic Community Health Study/Study of Latinos. Chest 155(1): 60-68.

8. Félix Amaro (2019) Paidopsiquiatria.cat.

9. Paul Carrillo-Mora, Jimena Ramírez-Peris, Katia Magaña-Vázquez (2019) Sleep neurobiology and its importance: Anthology for the university student. Journal of the Faculty of Medicine (Mexico).

10. Juan Carlos Sierra, Carlos Delgado-Domínguez \& Hugo Carretero-Dios (2019) Influence of the quality of sleep on psychopathological variables: A cross-examination between workers subjected to shifts and workers with normal hours. 